\title{
Estimates on Initial Coefficients of Certain Subclasses of Bi-Univalent Functions Associated with Quasi-Subordination
}

\author{
Amol B. Patil ${ }^{1 *}$ and Uday H. Naik ${ }^{2}$ \\ ${ }^{1}$ Department of First Year Engineering, AISSMS's, College of Engineering, Pune-411001, India \\ ${ }^{2}$ Department of Mathematics, Willingdon College, Sangli-416415, India \\ *Corresponding author E-mail:amol223patil@yahoo.co.in
}

\begin{abstract}
In the present investigation we introduce some subclasses of the function class $\Sigma$ of bi-univalent functions defined in the open unit disk $\mathbb{U}$, which are associated with the quasi-subordination. We obtain the estimates on initial coefficients $\left|a_{2}\right|$ and $\left|a_{3}\right|$ for the functions in these
\end{abstract} subclasses. Also several related subclasses are considered and connection with some known results are established.

Keywords: Analytic function; Bi-univalent function; Quasi-subordination; Subordination; Univalent function.

\section{Introduction}

Let $\mathscr{A}$ be the class of all analytic functions $f$ which are : (i) normalized by the conditions $f(0)=0$ and $f^{\prime}(0)=1$ and (ii) defined on the open unit disk $\mathbb{U}=\{z: z \in \mathbb{C},|z|<1\}$. The Taylor's series expansion of $f \in \mathscr{A}$ is

$f(z)=z+\sum_{k=2}^{\infty} a_{k} z^{k}$

The class of all functions in $\mathscr{A}$ which are univalent in the open unit disk $\mathbb{U}$ is denoted by $\mathscr{S}$. These univalent functions are invertible but their inverse functions may not be defined on the entire unit disk $\mathbb{U}$. The Koebe one-quarter theorem (see [4]) ensures that the image of $\mathbb{U}$ under every function $f \in \mathscr{S}$ contains a disk of radius $1 / 4$. Thus every function $f \in \mathscr{S}$ has an inverse (say $g$ ), satisfying $g(f(z))=z$ for all $z \in \mathbb{U}$ and $f(g(w))=w$, where $|w|<r_{0}(f), r_{0}(f) \geq 1 / 4$. In fact, it can be easily verified that the inverse function $g$ is given by

$g(w)=w-a_{2} w^{2}+\left(2 a_{2}^{2}-a_{3}\right) w^{3}-\left(5 a_{2}^{3}-5 a_{2} a_{3}+a_{4}\right) w^{4}+\cdots$.

A function $f \in \mathscr{A}$ is said to be bi-univalent in $\mathbb{U}$ if both $f$ and $f^{-1}$ are univalent in $\mathbb{U}$. The class of all bi-univalent functions defined in $\mathbb{U}$ is denoted by $\Sigma$.

Lewin [8] investigated the class $\Sigma$ of bi-univalent functions and showed that $\left|a_{2}\right|<1.51$ for the functions in the class $\Sigma$. Later, Brannan and Clunie [2] conjectured that $\left|a_{2}\right| \leq \sqrt{2}$. Also, Netanyahu [11] proved that $\max _{f \in \Sigma}\left|a_{2}\right|=4 / 3$. Still the coefficient estimate problem is open for each $\left|a_{n}\right|,(n=3,4, \cdots)$.

Brannan and Taha [3] (see also [17]) introduced certain subclasses of the bi-univalent function class $\Sigma$ similar to the subclasses $\mathscr{S}^{*}(\alpha)$ and $\mathscr{K}(\alpha)$ of starlike and convex functions of order $\alpha(0<\alpha \leq 1)$ respectively. Sirvastava et al.[16] introduced and investigated certain subclasses of bi-univalent function class $\Sigma$ and also obtained the initial coefficient bounds.
Ma and Minda [9] introduced the classes:

$$
\mathscr{S}^{*}(\phi)=\left\{f \in \mathscr{S} ;\left[z f^{\prime}(z) / f(z)\right] \prec \phi(z)\right\}
$$

and

$$
\mathscr{K}(\phi)=\left\{f \in \mathscr{S} ;\left[1+\left(z f^{\prime \prime}(z) / f^{\prime}(z)\right)\right] \prec \phi(z)\right\},
$$

where $\phi$ be an analytic function with positive real part in the unit disk $\mathbb{U}, \phi(0)=1, \phi^{\prime}(0)>0$ and maps $\mathbb{U}$ onto a region which is starlike with respect to 1 and symmetric with respect to the real axis. These classes includes several well known subclasses of starlike and convex functions respectively as special cases.

Robertson [15] introduced the concept of quasi-subordination in 1970. An analytic function $f$ is quasi-subordinate to another analytic function $\phi$, written as

$f(z) \prec_{q} \phi(z) ; \quad(z \in \mathbb{U})$

if there are the analytic functions $\psi$ and $w$ with $|\psi(z)| \leq 1, w(0)=$ 0 and $|w(z)|<1$ such that $f(z)=\psi(z) \phi(w(z))$. Observe that if $\psi(z)=1$ then $f(z)=\phi(w(z))$, so that $f(z) \prec \phi(z)$ in $\mathbb{U}$. (See [10] and [14] for work related to quasi-subordination.)

In this investigation we assumed that:

$\psi(z)=A_{0}+A_{1} z+A_{2} z^{2}+\cdots ; \quad(|\psi(z)| \leq 1, z \in \mathbb{U})$

and $\phi(z)$ is an analytic function in $\mathbb{U}$ with the form:

$\phi(z)=1+B_{1} z+B_{2} z^{2}+\cdots ; \quad\left(B_{1}>0\right)$.

\section{Coefficient Estimates for the Function Class $\mathscr{R}_{\Sigma}^{q}(\lambda, \phi)$}

Definition 2.1: A function $f \in \Sigma$ given by (1) is said to be in the class $\mathscr{R}_{\Sigma}^{q}(\lambda, \phi)$ if the following quasi-subordination holds:

$$
\left[(1-\lambda) \frac{f(z)}{z}+\lambda f^{\prime}(z)-1\right] \prec_{q}(\phi(z)-1)
$$


and

$\left[(1-\lambda) \frac{g(w)}{w}+\lambda g^{\prime}(w)-1\right] \prec_{q}(\phi(w)-1)$

where $z, w \in \mathbb{U}, \lambda \geq 1$ and the functions $g$ and $\phi$ are given by (2) and (5) respectively.

Theorem 2.2: Let $f(z)$ given by (1) be in the class $\mathscr{R}_{\Sigma}^{q}(\lambda, \phi)$. Then,

$\left|a_{2}\right| \leq \min \left\{\frac{\left|A_{0}\right| B_{1}}{1+\lambda}, \sqrt{\frac{\left|A_{0}\right|\left(B_{1}+\left|B_{2}-B_{1}\right|\right)}{1+2 \lambda}}\right\}$

and

$$
\begin{aligned}
\left|a_{3}\right| \leq \min \{ & \frac{\left(\left|A_{0}\right|+\left|A_{1}\right|\right) B_{1}}{1+2 \lambda}+\frac{A_{0}^{2} B_{1}^{2}}{(1+\lambda)^{2}}, \\
& \left.\frac{\left|A_{1}\right| B_{1}+\left|A_{0}\right|\left(B_{1}+\left|B_{2}-B_{1}\right|\right)}{1+2 \lambda}\right\} .
\end{aligned}
$$

Proof: Since $f \in \mathscr{R}_{\Sigma}^{q}(\lambda, \phi)$, there exist two analytic functions $u, v: \mathbb{U} \rightarrow \mathbb{U}$ with $u(0)=v(0)=0,|u(z)|<1,|v(w)|<1$ and a function $\psi$ defined by (4) satisfies:

$\left[(1-\lambda) \frac{f(z)}{z}+\lambda f^{\prime}(z)-1\right]=\psi(z)[\phi(u(z))-1]$

and

$\left[(1-\lambda) \frac{g(w)}{w}+\lambda g^{\prime}(w)-1\right]=\psi(w)[\phi(v(w))-1]$.

Define the functions $p$ and $q$ such that:

$p(z)=\frac{1+u(z)}{1-u(z)}=1+c_{1} z+c_{2} z^{2}+\cdots$

and

$q(w)=\frac{1+v(w)}{1-v(w)}=1+d_{1} w+d_{2} w^{2}+\cdots$

equivalently,

$u(z)=\frac{p(z)-1}{p(z)+1}=\frac{1}{2}\left[c_{1} z+\left(c_{2}-\frac{c_{1}^{2}}{2}\right) z^{2}+\cdots\right]$

and

$v(w)=\frac{q(w)-1}{q(w)+1}=\frac{1}{2}\left[d_{1} w+\left(d_{2}-\frac{d_{1}^{2}}{2}\right) w^{2}+\cdots\right]$.

Clearly $p$ and $q$ are analytic in $\mathbb{U}$ with $p(0)=q(0)=1$ and have their positive real part in $\mathbb{U}$. Hence $\left|c_{i}\right| \leq 2$ and $\left|d_{i}\right| \leq 2$ (see [12]). Using (10) and (11) together with (4) and (5) in the RHS of (8) and (9), we get

$$
\begin{array}{r}
\psi(z)[\phi(u(z))-1]=\frac{1}{2} A_{0} B_{1} c_{1} z+ \\
\left\{\frac{1}{2} A_{1} B_{1} c_{1}+\frac{1}{2} A_{0} B_{1}\left(c_{2}-\frac{c_{1}^{2}}{2}\right)+\frac{A_{0} B_{2}}{4} c_{1}^{2}\right\} z^{2}+\cdots
\end{array}
$$

and

$$
\begin{array}{r}
\psi(w)[\phi(v(w))-1]=\frac{1}{2} A_{0} B_{1} d_{1} w+ \\
\left\{\frac{1}{2} A_{1} B_{1} d_{1}+\frac{1}{2} A_{0} B_{1}\left(d_{2}-\frac{d_{1}^{2}}{2}\right)+\frac{A_{0} B_{2}}{4} d_{1}^{2}\right\} w^{2}+\cdots
\end{array}
$$

Since the function $f$ and its inverse $g$ are given by (1) and (2) respectively, we have

$$
\left[(1-\lambda) \frac{f(z)}{z}+\lambda f^{\prime}(z)-1\right]=(1+\lambda) a_{2} z+(1+2 \lambda) a_{3} z^{2}+\cdots
$$

and

$$
\begin{array}{r}
{\left[(1-\lambda) \frac{g(w)}{w}+\lambda g^{\prime}(w)-1\right]=-(1+\lambda) a_{2} w+} \\
(1+2 \lambda)\left(2 a_{2}^{2}-a_{3}\right) w^{2}+\cdots .
\end{array}
$$

Using (12) to (15) in (8) and (9) and then comparing the coefficients of $z, z^{2}, w$ and $w^{2}$; we get

$(1+\lambda) a_{2}=\frac{1}{2} A_{0} B_{1} c_{1}$,

$(1+2 \lambda) a_{3}=\frac{1}{2} A_{1} B_{1} c_{1}+\frac{1}{2} A_{0} B_{1}\left(c_{2}-\frac{c_{1}^{2}}{2}\right)+\frac{A_{0} B_{2}}{4} c_{1}^{2}$,

$-(1+\lambda) a_{2}=\frac{1}{2} A_{0} B_{1} d_{1}$

and

$$
(1+2 \lambda)\left(2 a_{2}^{2}-a_{3}\right)=\frac{1}{2} A_{1} B_{1} d_{1}+\frac{1}{2} A_{0} B_{1}\left(d_{2}-\frac{d_{1}^{2}}{2}\right)+\frac{A_{0} B_{2}}{4} d_{1}^{2}
$$

From (16) and (18), it follows that

$c_{1}=-d_{1}$

and

$8(1+\lambda)^{2} a_{2}^{2}=A_{0}^{2} B_{1}^{2}\left(c_{1}^{2}+d_{1}^{2}\right)$.

Also by adding (17) in (19) in light of (20), we get

$8(1+2 \lambda) a_{2}^{2}=2 A_{0} B_{1}\left(c_{2}+d_{2}\right)+A_{0}\left(B_{2}-B_{1}\right)\left(c_{1}^{2}+d_{1}^{2}\right)$.

Applying $\left|c_{i}\right| \leq 2$ and $\left|d_{i}\right| \leq 2$ in (21) and (22), we get the desired result (6).

Next, for the bound on $\left|a_{3}\right|$, by subtracting (19) from (17), we obtain

$a_{3}=a_{2}^{2}+\frac{2 A_{1} B_{1} c_{1}+A_{0} B_{1}\left(c_{2}-d_{2}\right)}{4(1+2 \lambda)}$.

Using (21) with $\left|c_{i}\right| \leq 2$ and $\left|d_{i}\right| \leq 2$ in (23), we get

$\left|a_{3}\right| \leq \frac{\left(\left|A_{0}\right|+\left|A_{1}\right|\right) B_{1}}{(1+2 \lambda)}+\frac{A_{0}^{2} B_{1}^{2}}{(1+\lambda)^{2}}$.

Also, using (22) with $\left|c_{i}\right| \leq 2$ and $\left|d_{i}\right| \leq 2$ in (23), we get

$\left|a_{3}\right| \leq \frac{\left|A_{1}\right| B_{1}+\left|A_{0}\right|\left(B_{1}+\left|B_{2}-B_{1}\right|\right)}{1+2 \lambda}$.

From (24) and (25), we get the desired result (7).

This completes the proof of Theorem 2.2.

Observe that, if we set $\psi(z)=1$ in Definition 2.1 , then the quasisubordination reduces to subordination and the subclass $\mathscr{R}_{\Sigma}^{q}(\lambda, \phi)$ reduces to $\mathscr{R}_{\Sigma}(\lambda, \phi)$. Hence we get the following corollary:

Corollary 2.3: Let the function $f(z)$ given by (1) be in the class $\mathscr{R}_{\Sigma}(\lambda, \phi)$. Then,

$\left|a_{2}\right| \leq \min \left\{\frac{B_{1}}{1+\lambda}, \sqrt{\frac{B_{1}+\left|B_{2}-B_{1}\right|}{1+2 \lambda}}\right\}$ 
and

$\left|a_{3}\right| \leq \min \left\{\frac{B_{1}}{1+2 \lambda}+\frac{B_{1}^{2}}{(1+\lambda)^{2}}, \frac{B_{1}+\left|B_{2}-B_{1}\right|}{1+2 \lambda}\right\}$.

If we set $\psi(z)=1$ and $\lambda=1$ in Theorem 2.2 , then we get the following corollary:

Corollary 2.4: Let the function $f(z)$ given by (1) be in the class $\mathscr{R}_{\Sigma}(\phi)$. Then,

$\mid a_{2} \leq \min \left\{\frac{B_{1}}{2}, \sqrt{\frac{B_{1}+\left|B_{2}-B_{1}\right|}{3}}\right\}$

and

$\left|a_{3}\right| \leq \min \left\{\frac{B_{1}}{3}+\frac{B_{1}^{2}}{4}, \frac{B_{1}+\left|B_{2}-B_{1}\right|}{3}\right\}$.

Remark 2.5: Corollaries (2.3) and (2.4) are the improvements of the estimates obtained in Theorem 2.1 given by Kumar et al. [7] and Theorem 2.1 given by Ali et al. [1], respectively.

Remark 2.6: If we set

$$
\begin{aligned}
\phi(z) & =\frac{1+(1-2 \beta) z}{1-z} \\
& =1+2(1-\beta) z+2(1-\beta) z^{2}+\cdots ; \quad(0 \leq \beta<1)
\end{aligned}
$$

in Corollaries (2.3) and (2.4) then we get the improvements of the estimates obtained in Theorem 3.2 given by Frasin and Aouf [5] and Theorem 2 given by Srivastava et al. [16], respectively.

3. Coefficient Estimates for the Function Class $\mathscr{S}_{\Sigma}^{*, q}(\phi)$

Definition 3.1: A function $f \in \Sigma$ given by (1) is said to be in the class $\mathscr{S}_{\Sigma}^{*, q}(\phi)$ if the following quasi-subordination holds:

$$
\left[\frac{z f^{\prime}(z)}{f(z)}-1\right] \prec_{q}(\phi(z)-1)
$$

and

$$
\left[\frac{w g^{\prime}(w)}{g(w)}-1\right] \prec_{q}(\phi(w)-1)
$$

where $z, w \in \mathbb{U}$ and the functions $g$ and $\phi$ are given by (2) and (5) respectively.

Theorem 3.2: Let $f(z)$ given by (1) be in the class $\mathscr{S}_{\Sigma}^{*, q}(\phi)$. Then,

$\left|a_{2}\right| \leq \min \{L, M, N\}$

where,

$$
\begin{gathered}
L=\sqrt{\left|A_{0}\right|\left(B_{1}+\left|B_{2}-B_{1}\right|\right)}, M=\sqrt{\frac{A_{0}^{2} B_{1}^{2}+\left|A_{0}\right|\left(B_{1}+\left|B_{2}-B_{1}\right|\right)}{2},} \\
N=\frac{\left|A_{0}\right| B_{1} \sqrt{\left|A_{0}\right| B_{1}}}{\sqrt{A_{0}^{2} B_{1}^{2}+\left|A_{0}\right|\left|B_{1}-B_{2}\right|}}
\end{gathered}
$$

and

$\left|a_{3}\right| \leq \min \{P, Q, R\}$

where,

$$
\begin{gathered}
P=\frac{\left|A_{1}\right| B_{1}}{2}+\left|A_{0}\right|\left(B_{1}+\left|B_{2}-B_{1}\right|\right), \\
Q=\frac{A_{0}^{2} B_{1}^{2}+\left|A_{0}\right|\left(B_{1}+\left|B_{2}-B_{1}\right|\right)-2\left|A_{1}\right| B_{1}}{2}, \\
R=\frac{1}{4}\left[\left(\left|A_{0}\right|+2\left|A_{1}\right|\right) B_{1}+3\left|A_{0}\right| B_{1} \cdot \max \left\{1,\left|\frac{B_{1}-4 B_{2}}{3 B_{1}}\right|\right\}\right] .
\end{gathered}
$$

Proof: Since $f \in \mathscr{S}_{\Sigma}^{*, q}(\phi)$, there exist two analytic functions $u, v$ : $\mathbb{U} \rightarrow \mathbb{U}$ with $u(0)=v(0)=0,|u(z)|<1,|v(w)|<1$ and a function $\psi$ defined by (4) satisfies:

$\left[\frac{z f^{\prime}(z)}{f(z)}-1\right]=\psi(z)[\phi(u(z))-1]$

and

$\left[\frac{w g^{\prime}(w)}{g(w)}-1\right]=\psi(w)[\phi(v(w))-1]$.

Define the functions $p$ and $q$ analytic in $\mathbb{U}$ as in (10) and (11) and then proceed similarly up to (13). Also on expanding LHS of (28) and (29) using (1) and (2), we get

$\left[\frac{z f^{\prime}(z)}{f(z)}-1\right]=a_{2} z+\left(2 a_{3}-a_{2}^{2}\right) z^{2}+\cdots$

and

$\left[\frac{w g^{\prime}(w)}{g(w)}-1\right]=-a_{2} w+\left(3 a_{2}^{2}-2 a_{3}\right) w^{2}+\cdots$.

Using (12), (13), (30) and (31) in (28) and (29) and then equating the coefficients of $z, z^{2}, w, w^{2}$; we get

$a_{2}=\frac{1}{2} A_{0} B_{1} c_{1}$,

$2 a_{3}=\frac{1}{2} A_{0} B_{1} c_{1} a_{2}+\frac{1}{2} A_{1} B_{1} c_{1}+\frac{1}{2} A_{0} B_{1}\left(c_{2}-\frac{c_{1}^{2}}{2}\right)+\frac{1}{4} A_{0} B_{2} c_{1}^{2}$,

$-a_{2}=\frac{1}{2} A_{0} B_{1} d_{1}$

and

$$
\begin{aligned}
4 a_{2}^{2}-2 a_{3}= & -\frac{1}{2} A_{0} B_{1} d_{1} a_{2}+\frac{1}{2} A_{1} B_{1} d_{1}+ \\
& \frac{1}{2} A_{0} B_{1}\left(d_{2}-\frac{d_{1}^{2}}{2}\right)+\frac{1}{4} A_{0} B_{2} d_{1}^{2}
\end{aligned}
$$

Using (32) and (34), we get

$c_{1}=-d_{1}$,

$8 a_{2}^{2}=\left(c_{1}^{2}+d_{1}^{2}\right) A_{0}^{2} B_{1}^{2}$

and

$4 a_{2}=\left(c_{1}-d_{1}\right) A_{0} B_{1}$

Adding (33) and (35) and then using (38), we get

$8 a_{2}^{2}=A_{0}\left[2\left(c_{2}+d_{2}\right) B_{1}+\left(c_{1}^{2}+d_{1}^{2}\right)\left(B_{2}-B_{1}\right)\right]$.

Adding (33) and (35) and then using (32) and (36), we get

$16 a_{2}^{2}=2 A_{0}^{2} B_{1}^{2} d_{1}^{2}+2\left(c_{2}+d_{2}\right) A_{0} B_{1}+A_{0}\left(c_{1}^{2}+d_{1}^{2}\right)\left(B_{2}-B_{1}\right)$.

Adding (33) and (35) and then using (37) and (38), we get

$4\left(A_{0}^{2} B_{1}^{2}+A_{0} B_{1}-A_{0} B_{2}\right) a_{2}^{2}=\left(c_{2}+d_{2}\right) A_{0}^{3} B_{1}^{3}$.

Now, (39), (40) and (41) along with $\left|c_{i}\right| \leq 2$ and $\left|d_{i}\right| \leq 2$, gives the desired estimate on $a_{2}$ as asserted in (26). 
Next, for estimate on $\left|a_{3}\right|$ subtracting (33) from (35) and then using (36), we get

$-4 a_{3}=-4 a_{2}^{2}+A_{1} B_{1} c_{1}+\frac{1}{2}\left(d_{2}-c_{2}\right) A_{0} B_{1}$.

Using (40) in (42), we get

$16 a_{3}=2 A_{0}^{2} B_{1}^{2} d_{1}^{2}+4 A_{0} B_{1} c_{2}+A_{0}\left(c_{1}^{2}+d_{1}^{2}\right)\left(B_{2}-B_{1}\right)-4 A_{1} B_{1} c_{1}$.

Subtracting (35) from (33) and then using (39), we get

$4 a_{3}=\frac{1}{2}\left(3 c_{2}+d_{2}\right) A_{0} B_{1}+c_{1}^{2} A_{0}\left(B_{2}-B_{1}\right)+A_{1} B_{1} c_{1}$

or

$4 a_{3}=\frac{1}{2} A_{0} B_{1} d_{2}+\frac{3 A_{0} B_{1}}{2}\left[c_{2}-\frac{2\left(B_{1}-B_{2}\right)}{3 B_{1}} c_{1}^{2}\right]+A_{1} B_{1} c_{1}$.

On applying the result given by Keogh and Merkes [6] (see also [13]), that is for any complex number $z,\left|c_{2}-z c_{1}^{2}\right| \leq 2 \cdot \max \{1,|2 z-1|\}$, along with $\left|d_{2}\right| \leq 2$ in (45), we obtain

$4\left|a_{3}\right| \leq\left|A_{0}\right| B_{1}+2\left|A_{1}\right| B_{1}+3\left|A_{0}\right| B_{1} \cdot \max \left\{1,\left|\frac{B_{1}-4 B_{2}}{3 B_{1}}\right|\right\}$.

Equations (43), (44) and (46) along with $\left|c_{i}\right| \leq 2$ and $\left|d_{i}\right| \leq 2$, gives the desired estimate on $a_{3}$ as asserted in (27) .

This completes the proof of Theorem 3.2.

Remark 3.3: If we set $\psi(z)=1$ and $\phi(z)=[1+(1-2 \beta) z] /(1-$ $z) ;(0 \leq \beta<1)$ in Theorem 3.2, then we have $B_{1}=B_{2}=2(1-\beta)$ and the class $\mathscr{S}_{\Sigma}^{*, q}(\phi)$ reduce to the class $\mathscr{S}_{\Sigma}^{*}(\beta)$ studied by Brannan and Taha [3]. Note that in the estimate of $a_{2}$ for the class $\mathscr{S}_{\Sigma}^{*}(\beta)$ we get an improvement in Theorem 3.1 given by Brannan and Taha [3]. Remark 3.4: If we set $\psi(z)=1$ and $\phi(z)=[(1+z) /(1-z)]^{\alpha} ;(0<$ $\alpha \leq 1)$ in Theorem 3.2, then we have $B_{1}=2 \alpha, B_{2}=2 \alpha^{2}$ and the class $\mathscr{S}_{\Sigma}^{*, q}(\phi)$ reduce to the class $\mathscr{S}_{\Sigma, \alpha}^{*}$ studied by Brannan and Taha [3]. Note that for the class $\mathscr{S}_{\Sigma, \alpha}^{*}$ we get the same estimate $\left|a_{2}\right| \leq 2 \alpha / \sqrt{1+\alpha}$ as in Theorem 2.1 given by Brannan and Taha [3].

\section{Coefficient Estimates for the Function Class} $\mathscr{K}_{\Sigma}^{q}(\phi)$

Definition 4.1: A function $f \in \Sigma$ given by (1) is said to be in the class $\mathscr{K}_{\Sigma}^{q}(\phi)$ if the following quasi-subordination holds:

$$
\left[\left(1+\frac{z f^{\prime \prime}(z)}{f^{\prime}(z)}\right)-1\right] \prec_{q}(\phi(z)-1)
$$

and

$\left[\left(1+\frac{w g^{\prime \prime}(w)}{g^{\prime}(w)}\right)-1\right] \prec_{q}(\phi(w)-1)$

where $z, w \in \mathbb{U}$ and the functions $g$ and $\phi$ are given by (2) and (5) respectively.

Theorem 4.2: Let $f(z)$ given by (1) be in the class $\mathscr{K}_{\Sigma}^{q}(\phi)$. Then,

$\left|a_{2}\right| \leq \min \left\{\sqrt{\frac{A_{0}^{2} B_{1}^{2}+\left|A_{0}\right|\left(B_{1}+\left|B_{2}-B_{1}\right|\right)}{6}}, \frac{\left|A_{0}\right| B_{1}}{2}\right\}$

and

$$
\begin{gathered}
\left|a_{3}\right| \leq \min \left\{\frac{A_{0}^{2} B_{1}^{2}+\left|A_{0}\right|\left(B_{1}+\left|B_{2}-B_{1}\right|\right)-\left|A_{1}\right| B_{1}}{6},\right. \\
\left.\frac{3 A_{0}^{2} B_{1}^{2}+2\left(\left|A_{0}\right|+\left|A_{1}\right|\right) B_{1}}{12}\right\} .
\end{gathered}
$$

Proof: Since $f \in \mathscr{K}_{\Sigma}^{q}(\phi)$, there exist two analytic functions $u, v$ : $\mathbb{U} \rightarrow \mathbb{U}$ with $u(0)=v(0)=0,|u(z)|<1,|v(w)|<1$ and a function $\psi$ defined by (4) satisfies:

$\left[\left(1+\frac{z f^{\prime \prime}(z)}{f^{\prime}(z)}\right)-1\right]=\psi(z)[\phi(u(z))-1]$

and

$\left[\left(1+\frac{w g^{\prime \prime}(w)}{g^{\prime}(w)}\right)-1\right]=\psi(w)[\phi(v(w))-1]$.

Proceeding similarly as in Theorem 2.2 and Theorem 3.2, we get

$$
\begin{array}{r}
2 a_{2} z+\left(6 a_{3}-4 a_{2}^{2}\right) z^{2}+\cdots=\frac{1}{2} A_{0} B_{1} c_{1} z+ \\
\left\{\frac{1}{2} A_{1} B_{1} c_{1}+\frac{1}{2} A_{0} B_{1}\left(c_{2}-\frac{c_{1}^{2}}{2}\right)+\frac{A_{0} B_{2}}{4} c_{1}^{2}\right\} z^{2}+\cdots
\end{array}
$$

and

$$
\begin{array}{r}
-2 a_{2} w+\left(8 a_{2}^{2}-6 a_{3}\right) w^{2}+\cdots=\frac{1}{2} A_{0} B_{1} d_{1} w+ \\
\left\{\frac{1}{2} A_{1} B_{1} d_{1}+\frac{1}{2} A_{0} B_{1}\left(d_{2}-\frac{d_{1}^{2}}{2}\right)+\frac{A_{0} B_{2}}{4} d_{1}^{2}\right\} w^{2}+\cdots .
\end{array}
$$

Equating the coefficients of $z, z^{2}$ in (51) and $w, w^{2}$ in (52), we get

$2 a_{2}=\frac{1}{2} A_{0} B_{1} c_{1}$,

$6 a_{3}=A_{0} B_{1} c_{1} a_{2}+\frac{1}{2} A_{1} B_{1} c_{1}+\frac{1}{2} A_{0} B_{1}\left(c_{2}-\frac{c_{1}^{2}}{2}\right)+\frac{A_{0} B_{2}}{4} c_{1}^{2}$,

$-2 a_{2}=\frac{1}{2} A_{0} B_{1} d_{1}$

and

$$
\begin{aligned}
\left(12 a_{2}^{2}-6 a_{3}\right)= & -A_{0} B_{1} d_{1} a_{2}+\frac{1}{2} A_{1} B_{1} d_{1}+ \\
& \frac{1}{2} A_{0} B_{1}\left(d_{2}-\frac{d_{1}^{2}}{2}\right)+\frac{A_{0} B_{2}}{4} d_{1}^{2}
\end{aligned}
$$

From (53) and (55), we get

$c_{1}=-d_{1}$,

$8 a_{2}=\left(c_{1}-d_{1}\right) A_{0} B_{1}$

and

$32 a_{2}^{2}=\left(c_{1}^{2}+d_{1}^{2}\right) A_{0}^{2} B_{1}^{2}$.

Adding (54) in (56) and then using (58) and (59), we get

$48 a_{2}^{2}=2 A_{0}^{2} B_{1}^{2} c_{1}^{2}+2\left(c_{2}+d_{2}\right) A_{0} B_{1}+A_{0}\left(c_{1}^{2}+d_{1}^{2}\right)\left(B_{2}-B_{1}\right)$.

Clearly, (58), (59) and (60) along with $\left|c_{i}\right| \leq 2$ and $\left|d_{i}\right| \leq 2$, yields the desired result (47).

Next, subtracting (54) from (56) and then using (57), we get

$-12 a_{3}=-12 a_{2}^{2}+\frac{1}{2}\left(d_{1}-c_{1}\right) A_{1} B_{1}+\frac{1}{2}\left(d_{2}-c_{2}\right) A_{0} B_{1}$.

Using (60) and (61), we get

$$
\begin{aligned}
48 a_{3}= & 2 A_{0}^{2} B_{1}^{2} c_{1}^{2}+4 A_{0} B_{1} c_{2}+ \\
& A_{0}\left(c_{1}^{2}+d_{1}^{2}\right)\left(B_{2}-B_{1}\right)-2\left(d_{1}-c_{1}\right) A_{1} B_{1} .
\end{aligned}
$$


Using (58) in (61), we get

$$
\begin{aligned}
-12 a_{3}= & \frac{1}{2}\left(d_{2}-c_{2}\right) A_{0} B_{1}+ \\
& \frac{1}{2}\left(d_{1}-c_{1}\right) A_{1} B_{1}-\frac{3\left(c_{1}-d_{1}\right)^{2} A_{0}^{2} B_{1}^{2}}{16} .
\end{aligned}
$$

Clearly, (62) and (63) along with $\left|c_{i}\right| \leq 2$ and $\left|d_{i}\right| \leq 2$, yields the desired result (48).

This completes the proof of Theorem 4.2.

Remark 4.3: If we set $\psi(z)=1$ and $\phi(z)=[1+(1-2 \beta) z] /(1-$ $z) ;(0 \leq \beta<1)$ in Theorem 4.2, then we have $B_{1}=B_{2}=2(1-\beta)$ and the class $\mathscr{K}_{\Sigma}^{q}(\phi)$ reduce to the class $\mathscr{K}_{\Sigma}(\beta)$ studied by Brannan and Taha [3]. Note that we get $\left|a_{2}\right| \leq 1-\beta$ and $\left|a_{3}\right| \leq(1-\beta)(3-$ $2 \beta) / 3$ for the functions in the class $\mathscr{K}_{\Sigma}(\beta)$, which is an improvement in Theorem 4.1 given by Brannan and Taha [3].

\section{References}

[1] R. M. Ali, S. K. Lee, V. Ravichandran and S. Supramaniam, "Coefficient estimates for bi-univalent Ma-Minda starlike and convex functions", Appl. Math. Lett., 25, (2012), 344-351.

[2] D. A. Brannan and J. G. Clunie (Eds.), Aspects of Contemporary Complex Analysis, (Proceedings of the NATO Advanced Study Institute held at the University of Durham, Durham; July 1-20, 1979), Academic Press, New York and London, (1980).

[3] D. A. Brannan and T. S. Taha, "On some classes of bi-univalent functions", Studia Univ. Babes-Bolyai Math, 31, (2), (1986), 70-77.

[4] P. L. Duren, Univalent Functions, Grundlehren der Mathematischen Wissenschaften, Springer, New York, (1983).

[5] B. A. Frasin and M. K. Aouf, "New subclasses of bi-univalent functions", Appl. Math. Lett., 24, (2011), 1569-1573.

[6] F. R. Keogh and E. P. Merkes, "A coefficient inequality for certain classes of analytic functions", Proc. Amer. Math. Soc., 20, (1969),

[7] S. S. Kumar, V. Kumar and V. Ravichandran, "Estimates for the initial coefficients of bi-univalent functions", Tamsui Oxf. J. Inf. and Math. Sci., 29, (4), (2013), 487-504.

[8] M. Lewin, "On a coefficient problem for bi-univalent functions", Proc. Amer. Math. Soc., 18, (1967), 63-68.

[9] W. C. Ma and D. Minda, "A unified treatment of some special classes of univalent functions", Proceedings of the Conference on Complex Analysis, Tianjin, (1992), 157-169.

[10] M. H. Mohd and M. Darus, "Fekete Szego problems for Quasisubordination classes", Abst. Appl. Anal., Article ID 192956, (2012),

[11] E. Netanyahu, "The minimal distance of the image boundary from the origin and the second coefficient of a univalent function in $|z|<1$ ", Arch. Rational Mech. Anal., 32, (1969), 100-112.

[12] Ch. Pommerenke, Univalent functions, Vandenhoeck and Rupercht, Göttingen, (1975).

[13] V. Ravichandran, Y. Polatoglu, M. Bolcal and A. Sen, "Certain subclasses of starlike and convex functions of complex order", Hacet. J. Math. Stat., 34, (2005), 9-15.

[14] F. Y. Ren, S. Owa and S. Fukui, "Some inequalities on quasisubordinate functions", Bull. Aust. Math. Soc., 43, (2), (1991), 317324 .

[15] M. S. Robertson, "Quasi-subordination and coefficient conjecture", Bull. Amer. Math. Soc., 76, (1970), 1-9.

[16] H. M. Srivastava, A. K. Mishra and P. Gochhayat, "Certain subclasses of analytic and bi-univalent functions", Appl. Math. Lett., 23, (2010), $1188-1192$

[17] T. S. Taha, Topics in Univalent Function Theory, Ph. D. Thesis, University of London, London, (1981). 